\title{
Characterization Report on Fuels for NEAMS Model Validation
}

Nuclear Technology

Research and Development

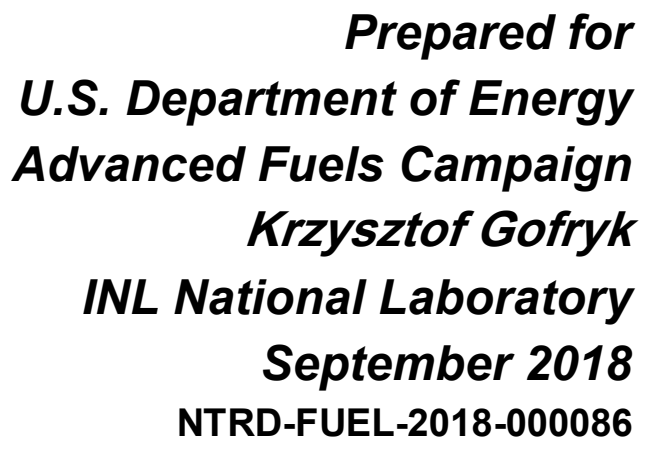




\section{DISCLAIMER}

This information was prepared as an account of work sponsored by an agency of the U.S. Government. Neither the U.S. Government nor any agency thereof, nor any of their employees, makes any warranty, expressed or implied, or assumes any legal liability or responsibility for the accuracy, completeness, or usefulness, of any information, apparatus, product, or process disclosed, or represents that its use would not infringe privately owned rights. References herein to any specific commercial product, process, or service by trade name, trade mark, manufacturer, or otherwise, does not necessarily constitute or imply its endorsement, recommendation, or favoring by the U.S. Government or any agency thereof. The views and opinions of authors expressed herein do not necessarily state or reflect those of the U.S. Government or any agency thereof. 


\section{ABSTRACT}

We have investigated the grain boundary scattering effect on the thermal transport behavior of uranium dioxide $\left(\mathrm{UO}_{2}\right)$. The polycrystalline samples having different grain sizes $(0.125,1.8$, and $7.2 \mu \mathrm{m})$ have been prepared by spark plasma sintering technique and characterized by $\mathrm{x}$-ray powder diffraction (XRD), scanning electron microscope (SEM), and Raman spectroscopy. The thermal transport properties (the thermal conductivity and thermoelectric power) have been measured in the temperature range $2-300 \mathrm{~K}$, and the results were analyzed in terms of various physical parameters contributing to the thermal conductivity in these materials in relation to grain size. We show that thermal conductivity decreases systematically with lowering grain size in the temperatures below $30 \mathrm{~K}$, where the boundary scattering dominates the thermal transport. At higher temperatures, more scattering processes are involved in the heat transport in these materials. We analyzed the results obtained using molecular dynamics (MD) calculations. 
INTENTIONALLY BLANK 


\section{CONTENTS}

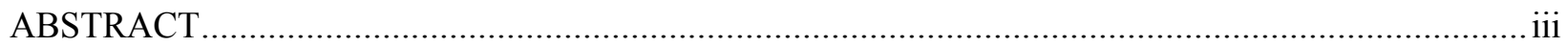

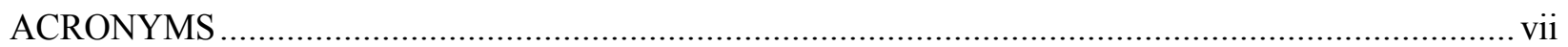

1. EFFECT OF GRAIN-BOUNDARY SCATTERING ON THE THERMAL CONDUCTIVITY OF URANIUM DIOXIDE .................................................................... 1

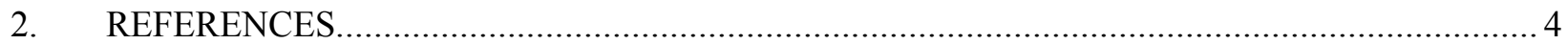

\section{FIGURES}

Figure 1. (a) XRD spectra show that the sintered pellets have $\mathrm{UO}_{2+\mathrm{x}}$ structure with ' $x$ ' values calculated by peak positions as shown in (b) for the high angle section. Superimposing feature of Raman spectra (c) indicates similar degree of interaction between defects and $\mathrm{UO}_{2}$ crystal structure in the sintered pellets.

Figure 2. Temperature dependence of the thermal conductivity of the $\mathrm{UO}_{2}$ polycrystalline samples having different grain sizes. The dotted line shows the thermal conductivity of $\mathrm{UO}_{2}$ single crystal.

Figure 3. The low temperature thermal conductivity of $\mathrm{UO}_{2}$ samples. The solid lines represent the least-square fits of the Callaway model to the experimental data; The grain size dependence (obtained from the Callaway model) of the low-temperature thermal conductivity peak (b), parameters B (c), D (d), and the mean free path of phonons L (e). The corresponding values for the $\mathrm{UO}_{2}$ single-crystal are displayed as a horizontal line in the relevant graphs. The dashed lines are guides to the eye. 
INTENTIONALLY BLANK 


\section{ACRONYMS}

MD molecular dynamics

SEM Scanning electron microscope

TD theoretical density

$\mathrm{T}_{\mathrm{N}} \quad$ Neel temperature

XRD $\quad \mathrm{x}$-ray diffraction 
INTENTIONALLY BLANK 


\section{CHARACTERIZATION REPORT ON FUELS FOR NEAMS MODEL VALIDATION}

\section{EFFECT OF GRAIN-BOUNDARY SCATTERING ON THE THERMAL CONDUCTIVITY OF URANIUM DIOXIDE}

Uranium dioxide is one of the most studied actinide materials as it is used as the primary fuel in commercial nuclear reactors [1][3]. There are around 500 active nuclear reactors, producing more than $15 \%$ of the total electricity worldwide. In a reactor, the heat energy produced from the nuclear fission events inside the fuel pellets is transformed into electricity. Thus, the heat transport mechanism, i.e., thermal conductivity of the fuel material, is an important parameter for fuel performance, regarding its efficiency and safety. A nuclear reactor operates at extreme environments that can include high temperature, high pressure, and high irradiation. As a result, a fuel pellet undergoes severe structural changes under irradiation conditions, including grain subdivision, fission gas bubble growth and redistribution, and extended defect accumulations [4][5] Thermal properties of the fuel material are greatly affected by these changes that ultimately affect the performance of a reactor. Numerous theoretical and experimental studies (see Refs. [6][9] and references therein) have been carried out to understand how these microstructure changes affect thermal transport properties of $\mathrm{UO}_{2}$. In an insulator, such as $\mathrm{UO}_{2}$, the lattice vibrations (phonons) responsible for the heat transport are scattered by different scattering centers, such as defects, grain boundaries, phonon-phonon, etc. Depending upon the temperature range, different scattering mechanisms dominate at different temperature regimes [10][12]. For instance, Umklapp phonon-phonon scattering dominates the thermal conductivity at high-temperatures, while the point-defect and boundary scattering govern the heat transport at intermediate and low temperatures, respectively. At low temperatures where the phonon mean free path is comparable to the grain-size, the grain boundary scattering mechanism is the main factor limiting the thermal conductivity. The effect of grain-size on the thermal conductivity has been investigated at low temperatures in other types of materials, such as semiconductors or thermoelectrics [13][17]. In the case of $\mathrm{UO}_{2}$, most of the studies on thermal properties are focused in the high temperature range (where the nuclear reactors operate) to better understand the fuel performance and reactor design [18][19].

Polycrystalline $\mathrm{UO}_{2}$ fuel pellets with three different grain-sizes $(0.125,1.8$, and $7.2 \mu \mathrm{m})$ were sintered by spark plasma sintering technique at Rensselaer Polytechnic Institute by Prof. Jie Lien and his research team. After the synthesis, several characterization techniques have been employed such as by x-ray diffraction (XRD), scanning electron microscope (SEM), and Raman spectroscopy (see Figure 1) to ensure sample quality with desired characteristics. All pellets were fully densified with measured density higher than 95\% theoretical density (TD). The XRD spectrum in Figure 1a and Figure 1b show that the sintered pellets are single phase $\mathrm{UO}_{2}$. Microstructure characterization was conducted by SEM microscopy, and the average grain sizes were estimated to be $0.125,1.8$, and $7.2 \mu \mathrm{m}$. Furthermore, the Raman spectroscopy (Figure 1c) shows the chemical bonding in those three different grain-sized samples are very similar, indicating comparable localized defect interaction with the crystal structure of $\mathrm{UO}_{2}$. The thermal transport properties (the thermal conductivity and thermoelectric power) have been measured in the temperature range 2-300 K, and the results were analyzed in terms of various physical parameters contributing to the thermal conductivity in these materials in relation to grain size. 
(a)

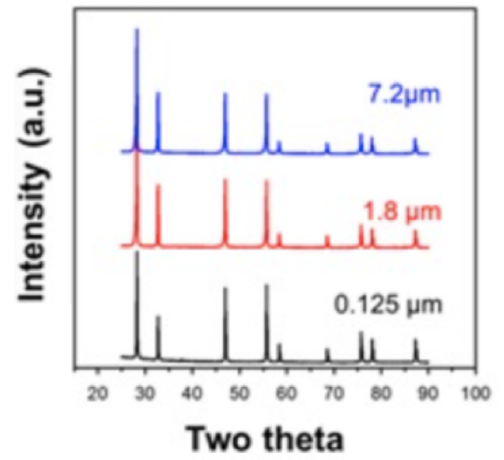

(b)

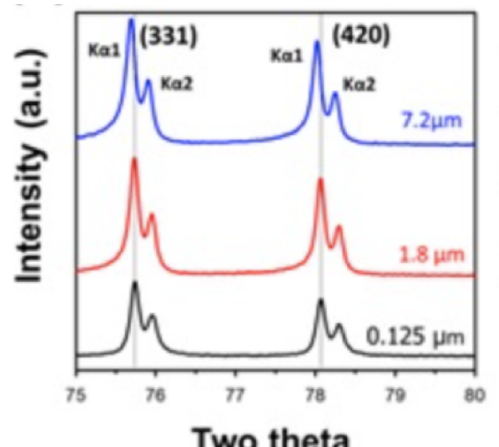

(c)

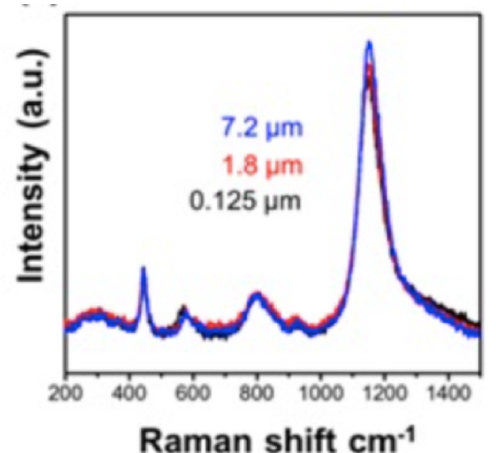

Figure 1. (a) XRD spectra show that the sintered pellets have $\mathrm{UO}_{2+\mathrm{x}}$ structure with ' $x$ ' values calculated by peak positions as shown in (b) for the high angle section. Superimposing feature of Raman spectra (c) indicates similar degree of interaction between defects and $\mathrm{UO}_{2}$ crystal structure in the sintered pellets.

Figure 2 shows the thermal conductivity, $\kappa$, of $\mathrm{UO}_{2}$ polycrystalline samples in the temperature range (2-300 K). For comparison, we have also included the temperature dependence of the thermal conductivity of $\mathrm{UO}_{2}$ single crystal [20]. The thermal conductivity of all samples shows similar temperature dependence as that of $\mathrm{UO}_{2}$ samples in the previous reports [20][22]. All $\kappa(\mathrm{T})$ curves consist of a broad maximum at $\mathrm{T}=220 \mathrm{~K}$ and a minimum at the Neel temperature, $\mathrm{T}_{\mathrm{N}}=30.8 \mathrm{~K}$. In addition, there is a well-defined peak at $\mathrm{T}=10 \mathrm{~K}$. Previous studies have revealed only a small difference in the thermal conductivity between single-crystal and polycrystalline $\mathrm{UO}_{2}$ [23]. At higher temperatures, the thermal conductivity of uranium dioxide single crystal is limited by 3-phonon Umklapp scattering processes together with resonant spin-phonon scattering [20][22]. These mechanisms are associated with a short mean free path, which may imply that grain boundaries have a relatively insignificant effect on the phonon conduction at this temperature range

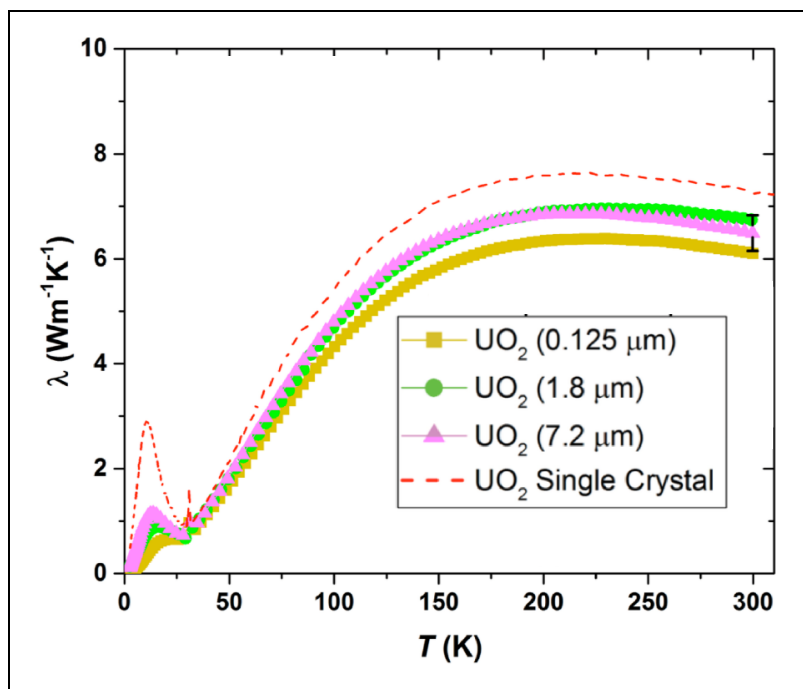

Figure 2. Temperature dependence of the thermal conductivity of the $\mathrm{UO}_{2}$ polycrystalline samples having different grain-sizes. The dotted line shows the thermal conductivity of $\mathrm{UO}_{2}$ single crystal. (see Refs. [10], [20], and [22]). As can be seen in the Figure 2, at room temperature, the thermal conductivity values of the $\mathrm{UO}_{2}$ polycrystalline samples vary slightly with grain size, and all the values are within $5 \%$ error of the measurement. These $\kappa(T)$ values may be also compared to the one obtained for single crystal $\mathrm{UO}_{2}$ material. As presented in the figure, the room temperature values of thermal conductivity of polycrystalline $\mathrm{UO}_{2}$ samples are $\sim 10 \%$ smaller than that of the single-crystal. Several possible scenarios besides grain boundary scattering may contribute to the reduction of the thermal conductivity in the polycrystalline samples as compared to single crystal, for instance, isotope effect and/or oxygen off-stoichiometry. The polycrystalline $\mathrm{UO}_{2}$ samples were synthesized using natural uranium isotope, whereas the $\mathrm{UO}_{2}$ single crystal consists of depleted uranium. The small percentage change of U-235 atoms, however, should have a negligible effect on the thermal conductivity value, much less than the observed $10 \%$ [24]. It has been shown that a much bigger impact 
on the thermal conductivity of uranium dioxide can be caused by the oxygen off-stoichiometry, i.e., $\mathrm{UO}_{2 \pm x}$. Theoretical studies have shown that both hyper- and hypo-stoichiometry lower the thermal conductivity of $\mathrm{UO}_{2}$ [25][26]. Also, from the previous experimental studies, it was observed that the thermal conductivity of $\mathrm{UO}_{2}$ is very sensitive to oxygen off-stoichiometry (not shown), and its value decreases as much as $30 \%$ for $\mathrm{UO}_{2.033}$, as compared to stoichiometric $\mathrm{UO}_{2}$ at room temperature. The variation of thermopower (not shown here) strongly suggests that very small oxygen off-stoichiometry might be present and play a role in lowering of the thermal conductivity in the $\mathrm{UO}_{2}$ samples, especially at high temperatures. To evaluate this important observation in more details, single crystal studies (on $\mathrm{UO}_{2}$ crystals with different oxygen content) are required.

Figure 3(a) shows the blown-up region of the thermal conductivity curves of the $\mathrm{UO}_{2}$ samples shown in Figure 2 in the range below $30 \mathrm{~K}$. As can be seen in this temperature range, the thermal conductivity decreases systematically with lowering the grain-size as expected. The variation of the thermal conductivity value $\kappa$-peak measured at the peak position near $\mathrm{T}=10 \mathrm{~K}$ (see the arrows in Figure 3(a) is shown in Figure 3(b). It is observed that the $\kappa$-peak increases systematically with the grain size as expected for grain boundary scattering and approaches the single crystal value (shown by the horizontal line) as the grain size is increased. This linear dependence of thermal conductivity with the grain size observed in $\mathrm{UO}_{2}$ is consistent with grain boundary scattering being a main source of the thermal resistance [20][22]. In order to get more information of how different scattering processes affect the thermal conductivity in uranium dioxide, we have used the Callaway model [27] to analyze the experimental data obtained. The thermal conductivity data were analyzed, and the variation of different parameters such as grain boundary (B), defects (D), or phonon mean-free path (L) with the grain size are presented in Figure 3(b-e).

In order to determine if changes in the measured thermal conductivity at high temperatures are due to the grain boundary Kapitza resistance, Prof. Michael Tonks' team (University of Florida) investigated this behavior using the analytical Kapitza resistance equation.
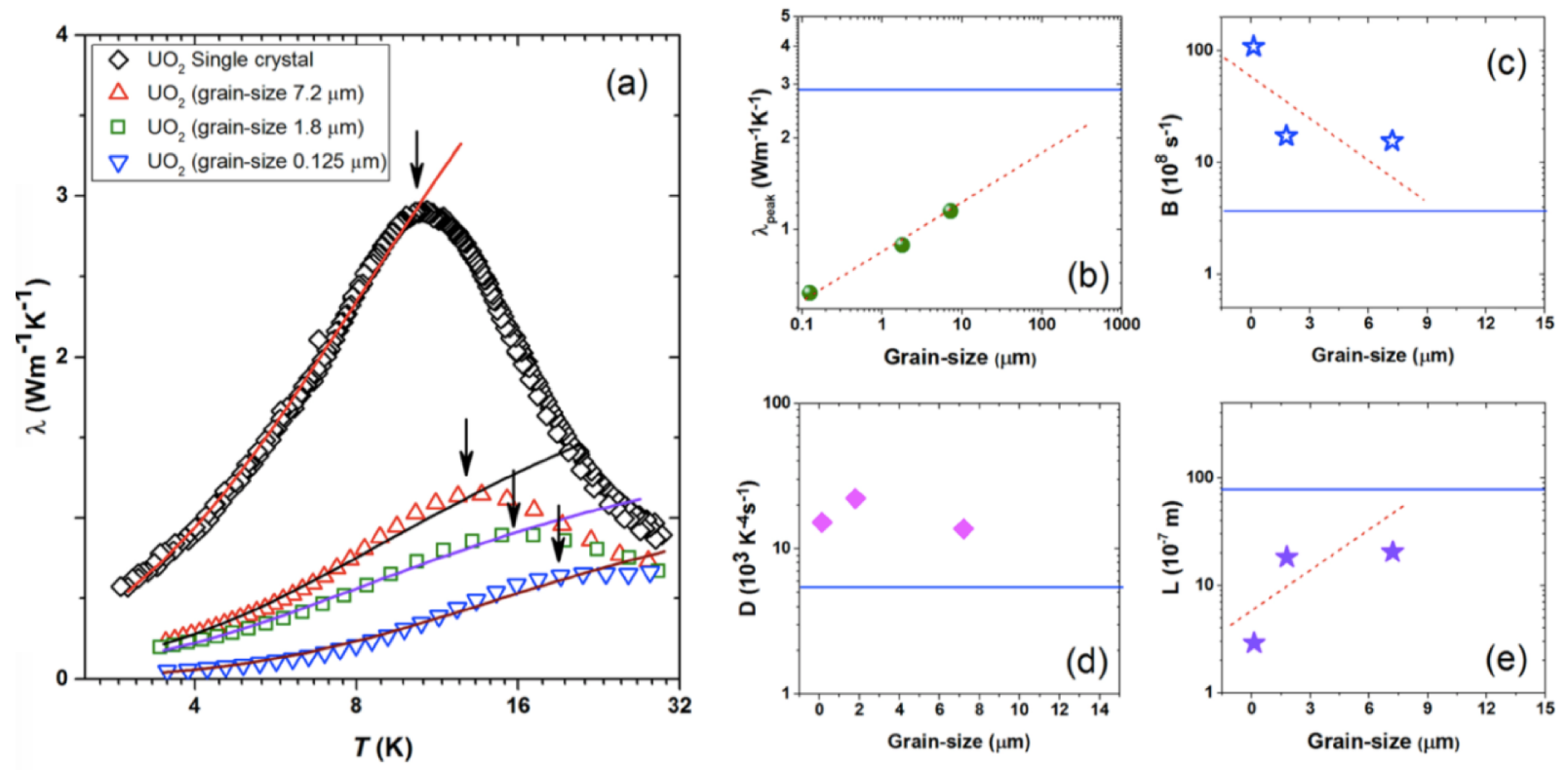

Figure 3. The low temperature thermal conductivity of $\mathrm{UO}_{2}$ samples. The solid lines represent the least-square fits of the Callaway model to the experimental data. The grain-size dependence (obtained from the Callaway model) of the low-temperature thermal conductivity peak (b), parameters B (c), D (d), and the mean free path of phonons $\mathrm{L}$ (e). The corresponding values for the $\mathrm{UO}_{2}$ single-crystal are displayed as a horizontal line in the relevant graphs. The dashed lines are guides to the eye. 
To summarize, we have synthesized $\mathrm{UO}_{2}$ samples having different grain size $(0.1251 .8$, and $7.2 \mu \mathrm{m})$ and investigated the grain size effect on thermal properties in this material. The samples have been characterized by x-ray powder diffraction (XRD), scanning electron microscope (SEM), and Raman spectroscopy. By performing low temperature thermal conductivity measurements, we have studied the grain boundary scattering related to grain size and its impact on thermal conductivity in this material. The thermal conductivity shows a systematic dependence on the grain size at low temperatures (below $30 \mathrm{~K}$ ) where the grain boundary and defect scatterings dominate the thermal transport. Such a behavior is not observed at higher temperatures due to other scattering processes that govern thermal conductivity of these materials, especially oxygen off-stoichiometry. The different scattering parameters, affecting the thermal conductivity, are estimated using the Callaway model. The grain boundary Kapitza resistance has been also evaluated by the molecular dynamics (MD) calculations. All the results obtained indicate importance of low temperature measurements to study grain boundary scattering in $\mathrm{UO}_{2}$. At higher temperatures, other scattering processes are involved in the heat transport in these materials which makes this analysis difficult. In addition, these studies point to the importance of oxygen off-stoichiometry for the thermal conductivity of $\mathrm{UO}_{2}{ }^{\mathrm{a}, \mathrm{b}}$.

\section{REFERENCES}

[1] K. L. Murty and I. Charit, An Introduction to Nuclear Materials: Fundamentals and Applications, Wiley, 2013.

[2] W. C. Patterson, Nuclear Power (second edition), Penguin Books, 1983.

[3] O. Runnalls, Uranium Dioxide - a Promising Nuclear Fuel (Atomic Energy of Canada Limited, 1958).

[4] R. J. M. Konings, T. Wiss, and O. Bene, Nat. Mater. 14, 47 (2015).

[5] E. Jin, C. Liu, and H. He, Int. Conf. Nuc. Eng. 1 (2016).

[6] S. Yamasaki, T. Arima, K. Idemitsu, and Y. Inagaki, Int. J. Thermophys. 28, 661 (2007).

[7] S. Motoyama, Y. Ichikawa, and Y. Hiwatari, Phys. Rev. B 60, 292 (1999).

[8] T. Watanabe, S. B. Sinnott, J. S. Tulenko, R. W. Grimes, P. K. Schelling, and S. R. Phillpot, J. Nuc. Mater. 375, 388 (2008).

[9] P. Ruello, G. Petot-Ervas, C. Petot, and L. Desgranges, J. Am. Ceram. Soc. 88, 604 (2005).

[10] T. M. Tritt, Thermal Conductivity Theory, Properties, and Applications, Vol. (Springer,2012).

[11] C. Kittel, Introduction to Solid State Physics, Vol. (Push Print Service, Delhi, 2006).

[12] D. Cahill, W. Ford, K. Goodson, G. Mahan, A. Majumdar, H. Maris, R. Merlin, and S. Phillpot, Appl. Phys. Rev. 93, 793 (2003).

[13] S. Yoon, O.-J. Kwon, S. Ahn, J.-Y. Kim, H. Koo, S.-H. Bae, J.-Y. Cho, J.-S. Kim, and C. Park, J. Elec. Mat. 42, 3390 (2013).

[14] J. Yang, G. P. Meisner, D. T. Morelli, and C. Uher, Phys. Rev. B 63, 014410 (2000).

[15] D. Spiteri, J. Anaya, and M. Kuball, J. Apl. Phys. 119, 085102 (2016).

a K. Shrestha, T. Yao, J. Lian, D. Antonio, M. Sessim, M. Tonks, and K. Gofryk, The grain-size effect on thermal conductivity of uranium dioxide - the paper is schedule to be submitted by the end of FY2018

b K. Gofryk, Effect of off-stoichiometry and grain size on thermal transport in uranium dioxide, talk at the Nuclear Materials conference NuMat 2018, Seattle, WA, 2018 
[16] J. C. Thompsonand B. A. Younglove, J. Phys. Chem. Solids 20, 146 (1961).

[17] X. Wang, Y. Yang, and L. Zhu, J. Appl. Phys. 110, 024312 (2011).

[18] T. Godfrey, W. Fulkerson, T. Kollie, J. Moore, and D. McElroy, J. Am. Ceram. Soc. 48, 298 (1965).

[19] T. Uchida, T. Sunaoshi, M. Kato, and K. Konashi, Prog. Nuc. Sci. and Tech. 2, 598 (2011).

[20] K. Gofryk, S. Du, C. Stanek, J. Lashley, X. Liu, R. Schulze, J. Smith, D. Safarik, D. Byler, K. McClellan, B. Uberuaga, B. Scott, and D. Andersson, Nat. Commun. 5, 4551 (2014).

[21] V. Haase, H. Keller-Rudek, L. Manes, B. Schulz, G. Schumacher, D. Vollath, and H. Zimmermann, Gmelin Handbook of Inorganic Chemistry, Vol. (Springer Verlag, 1986).

[22] J. P. Moore and D. L. McElroy, J. Am. Ceram. Soc. 54, 40 (1971).

[23] Thermal Conductivity of Uranium Dioxide, Technical Reports Series No. 59, IAEA, Vienna, 1966.

[24] G. A. Slack, Phys. Rev. 105, 829 (1957).

[25] X.-Y. Liu, M. D. Cooper, K. McClellan, J. Lashley, D. Byler, B. Bell, R. Grimes, C. R. Stanek, and D. A. Andersson, Summary report on UO2 thermal conductivity model refinement and assessment studies., Vol. (Report LA-UR-16-28212, Los Alamos National Laboratory, Los Alamos, NM, 2017).

[26] S. Nichenko and D. Staicu, J. Nucl. Mater. 433, 297 (2013).

[27] J. Callaway, Phys. Rev. 113, 1046 (1959). 